\title{
Inflamação e associação com parâmetros clínicos e nutricionais de pacientes em hemodiálise
}

\author{
Inflammation and association with clinical and nutritional parameters of patients on hemodialysis
}

\author{
Gilvan Campos Sampaio ${ }^{a}$ Antônia Caroline Diniz Brito ${ }^{a}$, Andreia de Jesus Ferreira Barrosa, \\ Elane Viana Hortegal ${ }^{b}$, Raimunda Sheyla Carneiro Dias ${ }^{c}$ \\ a Nutricionista. Residente do Programa de Residência Multiprofissional do Hospital Universitário da UFMA, Universidade Federal do Maranhão. \\ b Nutricionista. Mestre em Saúde Coletiva pela Universidade Federal do Maranhão. Departamento de Ciências Fisiológicas. Curso de Nutrição. \\ c Nutricionista. Serviço de Nefrologia do Hospital Universitário da Universidade Federal do Maranhão. \\ Fontes de financiamento: Fundação de Amparo à Pesquisa e Desenvolvimento Científico do Maranhão (FAPEMA).
}

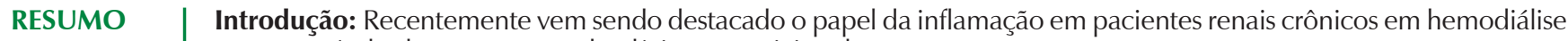
e sua possível relação com estado clínico e nutricional.

Objetivo: Avaliar a associação entre inflamação e parâmetros clínicos e nutricionais de pacientes em hemodiálise.

Materiais e Métodos: Estudo transversal. Foram coletados dados demográficos, clínicos e nutricionais: idade; Kt/ $\mathrm{V}$; níveis séricos de creatinina e vitamina D; índice de massa corporal (IMC); circunferências da cintura (CC), quadril (CQ) e muscular do braço (CMB); prega cutânea tricipital (PCT); diâmetro abdominal sagital (DAS) e força de preensão manual (FPM). Para avaliação do estado inflamatório utilizou-se o Malnutrition Inflammation Score (MIS). Para comparação entre as médias das variáveis clínicas e nutricionais com MIS foi realizado o teste t de Student pareado.

Resultados: Dos 73 pacientes avaliados, 54,2\% era do sexo masculino, com média de idade de 44,5 $\pm 15,4$ anos. $81,9 \%$ tinham menos de 60 anos, eram negros/pardos (95,8\%), com menos de 9 anos de estudo (70,4\%). Observou-se grande variação na classificação de desnutrição $(11,1$ a $66,7 \%)$, prevalência de inflamação $(57,7 \%)$, baixo risco cardiovascular (60,6\%) pela CC; $52,9 \%$ pela RCQ, sem excesso de gordura visceral (84,7\% - DAS). Níveis séricos adequados de creatinina e vitaminas D (69,4 e 83,8\%, respectivamente) foram encontrados. Foi observada associação do estado inflamatório com idade, creatinina, vitamina D e $\operatorname{FPM}(p<0,05)$.

Conclusão: Foi observada associação significativa do MIS com idade, creatinina, vitamina D e força muscular.

Palavras-chave: avaliação nutricional; diálise renal; inflamação.

Introduction: Recently, the role of inflammation in chronic renal failure patients on hemodialysis and its relation to clinical and nutritional status has been highlighted.

Objective: To evaluate the association between inflammation with clinical and nutritional parameters in hemodialysis patients.

Materials and Methods: Cross-sectional study. Demographic, clinical and nutritional data have been collected: age; Kt/V; serum creatinine and vitamin D; body mass index (BMI); waist circumference (WC), hip circumference (HC) and arm muscle circunference (AMC); tricipital skin fold (TSF); sagittal abdominal diameter (SAD) and hand grip strengh (HGS). For the evaluation of the inflammatory state the malnutrition inflammation score (MIS) was used. The paired Student's $t$ test was used to compare the means of clinical and nutritional variables with MIS.

Results: Of the 73 patients evaluated, $54.17 \%$ were male, with a mean age of $44.5 \pm 15.40$ years. $81.95 \%$ had less than 60 years, were black/brown (95.83\%), with less than 9 years of education (70.40\%). There was a wide variation in the classification of malnutrition (11.11\% to 66.66\%), prevalence of inflammation (57.75\%), low cardiovascular risk (60.56\%) by WC; $52.86 \%$ by WHR without excess of visceral fat (84.70\% - SAD). Appropriate serum creatinine and vitamin D (69.40 and $83.82 \%$, respectively) were found. It was observed an association between the inflammatory state, age, creatinine, vitamin D and HGS ( $p<0.05)$.

Conclusion: A significant association of MIS with age, creatinine, vitamin D and muscle strength was observed.

Keywords: nutritional assessment; hemodialysis; inflammation. 


\section{INTRODUÇÃO}

São muitas as implicações relacionadas à desnutrição proteico-energética (DEP) na progressão da doença renal crônica (DRC), em especial naqueles submetidos à hemodiálise (HD), levando ao aumento da morbimortalidade ${ }^{1}$.

Diversos fatores estão envolvidos na gênese da DEP na DRC: diminuição no consumo de energia e proteína, aumento do gasto energético, acidose metabólica, diminuição da atividade física, doenças associadas e o próprio procedimento dialítico. Além da DEP, alguns estudos vêm destacando o papel da inflamação nesses pacientes, o que acaba contribuindo para o agravamento do estado nutricional e maior morbimortalidade ${ }^{1,2}$.

Entre as causas da inflamação na hemodiálise destacam-se o próprio procedimento dialítico e fatores não relacionados à diálise, como a presença de comorbidades, intercorrências clínicas, fatores genéticos e a dieta ${ }^{3}$. O aumento deste estado inflamatório, caracterizado por níveis séricos elevados de proteína-C reativa (PCR) e citocinas pró-inflamatórias, inibem o apetite e causam aumento do catabolismo protéico, da lipólise e do gasto energético, impactando negativamente no estado nutricional desses pacientes ${ }^{4}$.

Devido à coexistência de inflamação e desnutrição em pacientes que fazem hemodiálise, a avaliação do estado de inflamação tem sido considerada um indicador útil no prognóstico nutricional ${ }^{5}$, bem como sua associação ao maior risco de morte nessa população ${ }^{1}$. Na avaliação do estado de inflamação a ferramenta Malnutrition Inflammation Score (MIS) têm sido estudada e validada na população em $\mathrm{HD}^{1,6}$. Resultados encontrados por diversos autores reforçam a aplicabilidade do MIS na predição do estado inflamatório de pacientes em HD, propondo ainda que o MIS substitua os níveis de interleucina-6 e de proteína C-reativa (PCR) na clínica diária para avaliar o estado inflamatório desses pacientes ${ }^{1,7,8}$.

A presença de inflamação leva a alterações dos compartimentos corporais. Alguns estudos têm mostrado uma possível associação do estado inflamatório de pacientes em HD com marcadores bioquímicos e antropométricos ${ }^{9,10,11}$, bem como com alguns parâmetros clínicos ${ }^{9}$. Considerando a relevância da inflamação no prognóstico do estado nutricional de pacientes em HD, o presente estudo tem como objetivo avaliar a associação entre parâmetros clínicos e nutricionais com a presença de inflamação em pacientes renais crônicos tratados com hemodiálise.

\section{MATERIAIS E MÉTODOS}

\section{Tipo de estudo}

Trata-se de um estudo de delineamento transversal e retrospectivo, realizado na Unidade Renal do Hospital
Universitário da Universidade Federal do Maranhão (HUUFMA). Este projeto faz parte de um projeto maior, intitulado "Estado Nutricional e funcionalidade de pacientes em tratamento dialítico, São Luís - MA" submetido à apreciação no Comitê de Ética em Pesquisa (CEP) do HUUFMA, atendendo as exigências da Resolução no 466/2012 do Conselho Nacional de Saúde.

\section{Fatores de inclusão e não inclusão}

Para constituição do grupo investigado, foram selecionados todos os pacientes em HD, há pelo menos 3 meses e com idade maior ou igual a 18 anos. Não foram incluídas gestantes, pessoas com amputação de membros, sequelas neurológicas, além daqueles com doenças autoimunes, infecciosas, câncer e síndrome da imunodeficiência adquirida. Outro motivo de não inclusão foi ausência de informações no prontuário. A amostra final deste estudo foi de 73 indivíduos, o que representa a $98,5 \%$ do total de pacientes que realizam o tratamento no referido serviço.

\section{Coleta de dados clínicos e nutricionais}

A coleta de dados foi realizada no Setor de Nefrologia, nos dias em que os pacientes estavam submetendo-se ao tratamento dialítico. Foram coletados dados sociais, demográficos, clínicos e laboratoriais a partir da consulta aos prontuários ou por meio de entrevistas com os pacientes, no período de junho a agosto de 2015. Os parâmetros clínicos e laboratoriais de interesse foram: adequação da diálise (Kt/V) (calculado através da fórmula de Daugirdas ${ }^{12}$; valor de referência $>1,2^{13}$ ), níveis séricos de creatinina (Metodologia Reação de Jaffé -- COBAS 6000 - ROCHE; valor de referência $\geq 10 \mathrm{mg} / \mathrm{dl}^{13}$ ) e vitamina $D$ (Metodologia Eletroquimioluminescência - COBAS 6000 - ROCHE; valor de referência $\geq 30 \mathrm{ng} / \mathrm{ml}$ ), obtidos no prontuário do paciente e com data mais próxima à avaliação nutricional.

As variáveis nutricionais foram obtidas a partir da avaliação antropométrica e de testes de função muscular, realizada conforme rotina do nutricionista do setor e incluíram: peso pós-dialítico, estatura, circunferências da cintura (CC), quadril (CQ) e muscular do braço (CMB), prega cutânea tricipital (PCT) diâmetro abdominal sagital (DAS) e a força de preensão manual (FPM), obtidos após o término da sessão de diálise e na segunda sessão da semana. O peso pós-dialítico, aferido em balança eletrônica (Filizola ${ }^{\circledR}$, Brasil), foi obtido do prontuário do paciente, efetuando-se a média dos registros de peso das quatro últimas sessões. Foi utilizada a média de quatro registros em função da variabilidade do estado de hidratação, que é peculiar a esses pacientes. A estatura foi obtida com o auxílio de um estadiômetro portátil 


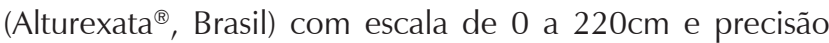
de $0,1 \mathrm{~cm}$.

O estado nutricional foi avaliado segundo o índice de massa corporal (IMC), obtido pela razão entre o peso pósdialítico e o quadrado da estatura, e adotada as classificações propostas pela Organização Mundial de Saúde ${ }^{14}$ para adultos e Lipchitz ${ }^{15}$ para idosos.

Para obtenção da CMB foram aferidas as medidas da circunferência do braço (CB) e PCT, conforme procedimentos descritos por Lohman et al. ${ }^{16}$. Ambas as medidas foram realizadas no braço oposto à fístula arteriovenosa. A CMB foi calculada conforme fórmula proposta por Frisancho ${ }^{17}$. Para a classificação do estado nutricional a partir da PCT e CMB foram calculados os respectivos percentuais de adequação tomando como referência a recomendação de Frisancho ${ }^{17,18}$.

Para avaliar a distribuição da gordura corporal foram aferidas a CC, CQ e DAS. A CC foi aferida no ponto médio entre a última costela e a crista-ilíaca, utilizando-se uma fita antropométrica flexível (Sanny ${ }^{\circledR}$, Brasil). A leitura foi realizada no momento final da expiração, tomando-se o cuidado para não haver compressão dos tecidos. Os valores obtidos foram comparados com os valores limítrofes $(\mathrm{CC}>94 \mathrm{~cm}$ para homens e CC $>80 \mathrm{~cm}$ mulheres) ${ }^{14}$. A CQ foi aferida com o paciente em pé, circundando o quadril na área de maior perímetro na altura dos glúteos, passando pela sínfise púbica, paralela ao chão. A relação cintura-quadril (RCQ), calculada pela razão entre a $\mathrm{CC}(\mathrm{cm})$ e a $\mathrm{CQ}(\mathrm{cm})$, teve o ponto de corte adotado valores $\geq 0,85$ para as mulheres e $\geq 1,00$ para os homens ${ }^{14}$.

A medida do DAS foi aferida com um caliper abdominal (Holtain Kahn Abdominal Caliper ${ }^{\circledR}$, British) de haste móvel e subdivisão de $0,1 \mathrm{~cm}$. Durante a avaliação, o paciente manteve-se na posição supina em uma mesa examinadora de superfície firme e com os joelhos flexionados. A medida foi tomada no ponto médio entre a última costela e a crista ilíaca. Os pontos de corte adotados foram: $\geq 23,1 \mathrm{~cm}$ para homens e $\geq 21,1 \mathrm{~cm}$ para mulheres ${ }^{19}$.

A avaliação funcional do músculo esquelético foi realizada por meio da medida da FPM. Na aferição foi utilizado o dinamômetro tipo Smedley $\left(\right.$ Jamar $\left.^{\circledR}\right)$, com escala de 0 a 100 kg e resolução de 1,0 kg, no braço oposto à fístula arteriovenosa. Para aqueles pacientes em uso de cateter, a aferição ocorreu no braço dominante. Os indivíduos receberam uma sequência clara de instruções quanto aos objetivos da aferição, sua posição durante o procedimento, número de aferições e intervalo de descanso entre as leituras e tiveram a oportunidade de testar o dinamômetro e ajustar a melhor empunhadura antes de realizar as leituras propriamente ditas. A aferição foi realizada com o paciente sentado e com braço elevado, formando um ângulo de $90^{\circ}$ com o antebraço. As medidas foram repetidas três vezes, adotando-se o maior valor alcançado. Foram considerados com força preservada valores $\geq 26 \mathrm{~kg}$ para homens e $\geq 16 \mathrm{~kg}$ para mulheres ${ }^{20}$.

\section{Estado de inflamação}

Após a coleta dos dados clínicos e nutricionais, os pacientes foram classificados segundo a presença ou não de sinais de inflamação, utilizando-se a ferramenta MIS1. Este escore apresenta 10 componentes divididos em quatro seções: história nutricional, exame físico, índice de massa corporal (IMC) e dados laboratoriais. Cada componente do MIS apresenta quatro níveis de severidade, pontuados de 0 (normal) a 3 (muito severo). A pontuação total varia de 0 (normal) a 30 (severamente desnutrido). Neste estudo, os indivíduos que apresentaram pontuação $\geq 6$ foram considerados com sinais de inflamação ${ }^{8}$.

\section{Análise Estatística}

Foi realizada análise descritiva para caracterização dos pacientes. As variáveis categóricas foram apresentadas por meio de frequências e porcentagens e as quantitativas por meio de média e desvio padrão (média \pm DP) ou mediana (P25-P75). A normalidade das variáveis foi avaliada pelo teste Shapiro-Wilk. Para comparação entre as médias das variáveis clínicas e nutricionais com MIS foi realizado o teste $\mathrm{t}$ de student pareado e o nível de significância adotado foi de $5 \%$. Os dados foram analisados pelo programa STATA 12.0 .

\section{RESULTADOS}

A amostra do estudo composta por 73 pacientes, com prevalência do sexo masculino $(54,2 \%)$. A média de idade foi de 44,5 $\pm 15,4$ anos, com predominância de indivíduos menores de 60 anos (81,9\%), negros e pardos $(95,8 \%)$ e com menos de 9 anos de estudo (70,4\%). Os parâmetros clínicos revelaram maior prevalência de adequação do Kt/v $(93,2 \%)$ e predomínio de pacientes com mais de 60 meses de HD $(47,2 \%)$ (Tabela 1$)$.

A avaliação do estado nutricional demonstrou uma maior prevalência de eutrofia (55,6\%), segundo IMC. No entanto, a adequação da PCT e CMB evidenciou desnutrição em 66,7 e $51,4 \%$ dos pacientes, respectivamente. Quanto aos parâmetros de distribuição da gordura corporal, a maioria dos investigados apresentaram valores adequados de CC $(60,6 \%)$, RCQ $(52,9 \%)$ e DAS $(84,7 \%)$. Valores séricos de creatinina e vitamina D estavam adequados em $69,4 \%$ e $83,8 \%$ dos pacientes, respectivamente. A média do MIS foi de $6,4 \pm 3,3 \mathrm{com}$ a maioria dos indivíduos apresentando sinais de inflamação $(57,7 \%)$ (Tabela 2$)$. 
Tabela 1. Características sócio demográficas e clínicas de pacientes submetidos à hemodiálise em um Hospital Universitário.

\begin{tabular}{lcc}
\hline \multicolumn{1}{c}{ Variáveis } & \multicolumn{2}{c}{ Total $(\mathbf{n}=\mathbf{7 3})$} \\
\cline { 2 - 3 } Sexo & $\mathbf{n}$ & $\%$ \\
$\quad$ Masculino & 39 & 54,2 \\
$\quad$ Feminino & 33 & 45,8 \\
Idade (anos) & & \\
$\quad<40$ & 29 & 40,3 \\
$\quad 40-60$ & 30 & 41,7 \\
$\quad>60$ & 13 & 18,1 \\
Cor da pele & & \\
$\quad$ Branco & 3 & 4,2 \\
$\quad$ Negros e pardos & 69 & 95,8 \\
Anos de estudo* & & \\
$\quad<9$ anos de estudo & 50 & 70,4 \\
Kt/ $>$ & & \\
$\quad<1,2$ & 5 & 6,9 \\
$\quad \geq 1,2$ & 67 & 93,0 \\
Tempo de hemodiálise & & \\
$\quad>3$ meses e $<12$ meses & 8 & 11,1 \\
$\geq 12$ meses e $<60$ meses & 30 & 41,7 \\
$\geq 60$ meses & 34 & 47,2 \\
\hline
\end{tabular}

${ }^{*} \mathrm{n}=71 ; \mathrm{Kt} / \mathrm{V}$ : eficiência dialítica.

Tabela 2. Características nutricionais e bioquímicas de pacientes submetidos à hemodiálise em um Hospital Universitário.

\begin{tabular}{|c|c|c|}
\hline \multirow{2}{*}{ Variáveis } & \multicolumn{2}{|c|}{ Total $(n=73)$} \\
\hline & n & $\%$ \\
\hline $\begin{array}{l}\mathrm{IMC}\left(\mathrm{kg} / \mathrm{m}^{2}\right) \\
\text { Eutrófico } \\
\text { Desnutrido } \\
\text { Sobrepeso/obesidade }\end{array}$ & $\begin{array}{c}40 \\
8 \\
24\end{array}$ & $\begin{array}{l}55,6 \\
11,1 \\
33,3\end{array}$ \\
\hline $\begin{array}{l}\text { \%PCT } \\
\text { Eutrofia } \\
\text { Desnutrição } \\
\text { Sobrepeso/Obesidade }\end{array}$ & $\begin{array}{l}11 \\
48 \\
13\end{array}$ & $\begin{array}{l}15,3 \\
66,7 \\
18,1\end{array}$ \\
\hline $\begin{array}{l}\text { \%CMB } \\
\text { Eutrofia } \\
\text { Desnutrição }\end{array}$ & $\begin{array}{l}35 \\
37\end{array}$ & $\begin{array}{l}48,6 \\
51,4\end{array}$ \\
\hline $\begin{array}{l}\mathrm{CC}(\mathrm{cm}) \\
\quad<80 \mathrm{~cm} \text { 우 } \mathrm{e}<94 \mathrm{~cm} \text { ᄋ } \\
\geq 80 \mathrm{~cm} \text { 우 } \mathrm{e} \geq 94 \mathrm{~cm} \text { o }^{7}\end{array}$ & $\begin{array}{l}43 \\
28\end{array}$ & $\begin{array}{l}60,6 \\
39,4\end{array}$ \\
\hline $\begin{array}{l}\text { RCQ } \\
<0,85 \text { 우 e }<1,00 \text { o } \\
\geq 0,85 \text { 우 } \mathrm{e} \geq 1,00 \text { 이 }\end{array}$ & $\begin{array}{l}37 \\
33\end{array}$ & $\begin{array}{l}52,9 \\
47,1\end{array}$ \\
\hline $\begin{array}{l}\text { DAS }(\mathrm{cm}) \\
\text { Baixo risco } \\
\text { Alto risco }\end{array}$ & $\begin{array}{l}61 \\
11\end{array}$ & $\begin{array}{l}84,7 \\
15,3\end{array}$ \\
\hline $\begin{array}{l}\text { MIS } \\
\text { Sem sinais de inflamação } \\
\text { Com sinais de inflamação }\end{array}$ & $\begin{array}{l}30 \\
41\end{array}$ & $\begin{array}{l}42,2 \\
57,7\end{array}$ \\
\hline $\begin{array}{l}\text { FPM }(\mathrm{kg}) \\
\text { Força preservada oำ 우 } \\
\text { Força reduzida } \sigma^{\top} \text { 우 }\end{array}$ & $\begin{array}{l}37 \\
34\end{array}$ & $\begin{array}{l}52,1 \\
47,9\end{array}$ \\
\hline $\begin{array}{l}\text { Creatinina }(\mathrm{mg} / \mathrm{dl}) \\
\quad \geq 10 \\
<10\end{array}$ & $\begin{array}{l}50 \\
22\end{array}$ & $\begin{array}{l}69,4 \\
30,6\end{array}$ \\
\hline $\begin{array}{l}\text { Vitamina } \mathrm{D}(\mathrm{ng} / \mathrm{dl})^{*} \\
\geq 30 \text { (normal) } \\
<30 \text { (abaixo da normalidade) }\end{array}$ & $\begin{array}{l}57 \\
11\end{array}$ & $\begin{array}{l}83,8 \\
16,2\end{array}$ \\
\hline
\end{tabular}

* $\mathrm{n}=68$; IMC: índice de massa corporal; \%PCT e \%CMB: porcentagem de adequação com relação ao percentil 50 da prega cutânea tricipital e circunferência muscular do braço; CC: circunferência da cintura; RCQ: razão cintura-quadril; DAS: diâmetro abdominal sagital; MIS: Malnutrition Inflammation Score; FPM: Força de preensão manual.
A Tabela 3, apresenta a associação das médias das variáveis demográficas, clínicas e nutricionais com a presença ou não de sinais de inflamação avaliado por meio do MIS. Foi observada associação significativa do MIS com idade, creatinina, vitamina D e FPM.

Tabela 3. Associação entre estado de inflamação e variáveis demográficas, clínicas e nutricionais de pacientes submetidos à hemodiálise em um Hospital Universitário.

\begin{tabular}{lccc}
\hline \multicolumn{1}{c}{ Variáveis } & $\begin{array}{c}\text { Sem sinais de } \\
\text { Inflamação }\end{array}$ & $\begin{array}{c}\text { Com sinais de } \\
\text { inflamação }\end{array}$ & Valor de $\boldsymbol{p}$ \\
\hline Idade (anos) & $30 \pm 11,9$ & $41 \pm 16,5$ & 0,006 \\
Kt/V & $1,5 \pm 0,3$ & $1,5 \pm 0,2$ & 0,618 \\
Creatinina (mg/dl) & $14,2(13,5-15,7)$ & $10,9(7,8-13,2)$ & 0,001 \\
Vitamina D (ng/dl) & $54,3 \pm 14,6$ & $46,6 \pm 14,6$ & 0,039 \\
CC $(\mathrm{cm})$ & $83,8 \pm 14,1$ & $85,7 \pm 10,4$ & 0,515 \\
RCQ & $0,89 \pm 0,1$ & $0,93 \pm 0,1$ & 0,545 \\
DAS (cm) & $18,72 \pm 3,6$ & $18,88 \pm 2,7$ & 0,829 \\
PCT (\%') & $100,6 \pm 64,8$ & $77,9 \pm 38,1$ & 0,067 \\
CMB (\%) & $90,7 \pm 12,5$ & $93 \pm 15,9$ & 0,521 \\
FPM (kg) & $22,9 \pm 8,9$ & $18,5 \pm 7,2$ & 0,028 \\
\hline
\end{tabular}

Kt/V: eficiência dialítica; CC: Circunferência da cintura; RCQ: Razão cintura/quadril; DAS Diâmetro abdominal sagital; PCT: Prega cutânea tricipital; CMB: Circunferência muscular do braço; FPM: Força de preensão manual; MIS: Malnutrition Inflammation Score.

\section{DISCUSSÃO}

A prevalência de indivíduos do sexo masculino, negros e pardos, bem como baixo nível de escolaridade parece ser uma tendência comum de pacientes em HD no Brasil ${ }^{9,21}$. Os resultados do Censo de diálise realizado pela Sociedade Brasileira de Nefrologia demonstraram que cerca de $32 \%$ dos pacientes em tratamento dialítico tinham idade superior a 65 anos $^{22}$. Diferente dos resultados do Censo de diálise, o presente estudo demonstrou que $18,1 \%$ dos pesquisados apresentavam idade $>60$ anos. Resultados semelhantes foram encontrados no estudo de Biavo et al. ${ }^{21}$. Com relação à adequação da diálise, o Kt/ $\mathrm{V}$ esteve dentro do recomendado em 93,1\% dos pacientes com média de $1,5 \pm 0,2$, dado importante já que, diálise insuficiente está associada ao acúmulo de toxinas urêmicas, o que contribui para alterações gastrointestinais, interferindo no apetite, causas de desnutrição ${ }^{13}$.

Os resultados do estudo demonstram uma grande variação na classificação do estado nutricional dos pacientes. Estas diferenças podem ser explicadas devido aos diferentes pontos de corte e técnicas de medição adotadas ${ }^{5,6}$, bem como alterações no estado de hidratação dos pacientes em $\mathrm{HD}^{23}$. Quando avaliados pelo IMC, prevaleceu a eutrofia, entretanto, a adequação da PCT e CMB revelou desnutrição. 
Na avaliação da função e massa muscular a maioria apresentou adequação de FPM e creatinina sérica $\geq 10 \mathrm{mg} / \mathrm{dl}$. Os indicadores nutricionais que avaliam a distribuição da gordura corporal (CC, RCQ e DAS) também demonstraram maior prevalência de eutrofia. A depleção dos estoques de gordura corporal anunciados pela PCT pode ter contribuído para o baixo acúmulo de gordura na região abdominal.

No estudo, quanto mais avançada a idade, maior a pontuação no MIS. Esta associação também foi evidenciada no estudo de Dantas et al. ${ }^{24}$, onde houve correlação positiva e moderada entre estado de inflamação e idade. Corroborando com nossos resultados, Barros et al. ${ }^{5}$ não só encontraram associação entre MIS e idade $(p<0,01)$, como também entre MIS e tempo de HD $(r=0,320, p<0,05)$. Apesar da não investigação da associação entre MIS e tempo de HD, é importante ressaltar que houve uma maior prevalência de pacientes com tempo de HD superior a cinco anos, sugerindo uma possível contribuição do tempo de tratamento no estado inflamatório.

Estudos prévios têm demostrado uma alta prevalência de deficiência de vitamina $D$ na população em geral, fato que se agrava em pacientes renais crônicos ${ }^{26}$. No presente estudo, os níveis séricos de vitamina D estiveram adequados em 83,8\% da população estudada. Todavia é necessária cautela na interpretação deste resultado, uma vez que essa prevalência pode estar relacionada à suplementação periódica de vitamina D, e não a ingestão adequada de fontes alimentares desta vitamina. Outra observação a ser feita é o fato de não terem sido avaliados os níveis de PTH, o que também pode ter influenciado os níveis séricos da vitamina nesses pacientes. Entretanto, foi observada associação entre os níveis séricos de vitamina $\mathrm{D}$ e sinais de inflamação $(p<0,05)$. Bucharles et $\mathrm{al}^{10}{ }^{10}$, estudando pacientes em HD que não receberam a terapia de vitamina $D$, encontraram que a inflamação foi mais prevalente naqueles com hipovitaminose D. Em outro estudo o mesmo autor Bucharles et al. ${ }^{27}$, agora estudando os efeitos da suplementação de colecalciferol via oral em grupo de pacientes com níveis de $25(\mathrm{OH}) \mathrm{D}<30 \mathrm{ng} / \mathrm{mL}$, observou uma redução significativa nos níveis de proteína C-reativa e interleucina-6, após 3 e 6 meses de suplementação.

Os resultados do estudo mostraram que a maioria dos entrevistados apresentou valores de creatinina $\geq 10 \mathrm{mg} / \mathrm{dl} \mathrm{e}$ foi possível observar associação significativa entre os valores de creatinina e sinais de inflamação $(p<0,05)$. Resultados semelhantes foram encontrados no estudo de Oliveira ${ }^{25}$, onde foi observado correlação significativa discreta a moderada do estado inflamatório avaliado através do MIS com creatinina. Essa associação já era esperada uma vez que a creatinina sérica reflete os estoques de proteína somática e redução nos níveis de creatinina sérica em pacientes em HD sugerem déficit de massa muscular, fato que é agravado na presença de sinais de inflamação $0^{5}$.

Marcadores inflamatórios mais elevados podem estimular a perda de aminoácidos e consequentemente a perda de proteínas das fibras musculares provocando efeitos adversos sobre a função muscular em pacientes em $\mathrm{HD}^{4,30}$. No presente estudo foi observado que o grupo de pacientes com sinais de inflamação teve menor média $(18,5 \pm 7,2 \mathrm{~kg})$ de força muscular quando comparados com o grupo sem sinais de inflamação $(22,9 \pm 8,9 \mathrm{~kg})$. Corroborando com nossos resultados Silva et al. ${ }^{6}$, estudando a função muscular de homens e mulheres em HD, também observaram uma função muscular diminuída avaliada pela FPM em pacientes mais inflamados (MIS > 6 pontos), após o ajuste por idade, raça, tempo de diálise, e Kt/ $\mathrm{V}$.

Como limitações do estudo podemos destacar o reduzido valor amostral, o que pode ter impossibilitado a identificação de diferenças estatisticamente significativas. Outra limitação é a ausência de análise laboratorial de marcadores considerados padrão-ouro para avaliação da inflamação como IL-6 e PCR. Como ponto positivo podemos destacar o uso da MIS como ferramenta simples para avaliar o estado de inflamação de pacientes em hemodiálise e o uso de métodos de avaliação nutricional (DAS e FPM) ainda pouco explorados na literatura nesta área.

Em conclusão, os resultados reforçam a aplicabilidade do MIS como ferramenta clínica na predição do estado inflamatório de pacientes em hemodiálise. A maior pontuação do instrumento esteve associada à idade, perda de massa e força muscular e menores níveis de vitamina D. Atenção especial deve ser dada aos pacientes de maior idade uma vez que, o estado inflamatório está associado com maiores médias de idade. No entanto mais estudos se fazem necessários para compreender o real papel do estado inflamatório sobre parâmetros clínicos e nutricionais de pacientes em hemodiálise.

\section{AGRADECIMENTOS}

Agradecemos aos pacientes e funcionários da Unidade Renal do Hospital Universitário da Universidade Federal do Maranhão - UFMA pela disponibilidade e à Fundação de Amparo à Pesquisa e Desenvolvimento Científico do Maranhão - FAPEMA pelo auxílio financeiro.

\section{REFERÊNCIAS}

1. Rambod M, Bross R, Zitterkoph J, Benner D, Pithia J, Colman S, Kovesdy CP, Kopple JD, Kalantar-Zadeh K. Association of MalnutritionInflammation Score with quality of life and mortality in hemodialysis patients: a 5-year prospective cohort study. Am J Kidney Dis. 2009;53(2):298-309. https://doi.org/10.1053/j. ajkd.2008.09.018 
2. Meuwese CL, Stenvinkel P, Dekker FW, Carrero JJ. Monitoring of inflammation in patientson dialysis: forewarned is forearmed. Nat. Rev. Nephrol. 2011;7:166-76. https://doi.org/10.1038/ nrneph.2011.2

3. Carrero JJ, Yilmaz MI, Lindholm B, Stenvinkel P. Cytokine dysregulation in chronic kidney disease: how can we treat it? Blood Purif. 2008;26(3):291-9. https://doi.org/10.1159/000126926

4. Stenvinkel P. Inflammation in end-stage renal failure: could it be trated? Nephrol Dial Transplant. 2002;17(8):33-8. https://doi. org/10.1093/ndt/17.suppl_8.33

5. Barros A, Oliboni AS, Felix R, Lucas LS, d'Avila DO. Hemodialysis patients: inflammatory status andleanbodymass. Sci Med. 2014;24(1):6-10. https://doi.org/10.15448/19806108.2014.1.14812

6. Silva LF, Matos CM, Lopes GB, Martins MT, Martins MS, Arias LU, Pisoni RL, Lopes AA. Handgrip strength as a simple indicator of possible malnutrition and inflammation in men and women on maintenance hemodialysis. J Ren Nutr. 2011;21(3):235-45. https:// doi.org/10.1053/j.jrn.2010.07.004

7. Afşar B, Sezer S, Ozdemir FN, Celik H, Elsurer R, Haberal M. Malnutrition-inflammation score is a useful tool in peritoneal dialysis patients. Perit Dial Int. 2006;26(6):705-11.

8. Demir M, Kucuk A, Sezer MT, Altuntas A, Kaya S. Malnutritioninflammationscore and endothelial dysfunction in hemodialysis patients. J Ren Nutr. 2010;20(6):377-83. https://doi.org/10.1053/j. jrn.2010.03.002

9. Prado LVS, Santos EMC, Luz MCL, Silva PC, Gadelha PCFP. Inflammation and malnutrition in renal replacement therapy unit of Northeastern Brazil. Nutr Clín Diet Hosp. 2014;34(3):29-36.

10. Bucharles S, Barberato SH, Stinghen AE, Gruber B, Meister H, Mehl A, Piekala L, Dambiski AC, Souza A, Olandoski M, Pecoits-Filho R. Hypovitaminosis D is associated with systemic inflammation and concentric myocardial geometric pattern in hemodialysis patients with low iPTH levels. Nephron Clin Pract. 2011;118(4):c384-91. https://doi.org/10.1159/000323664

11. Delatim FC. Associações entre marcadores nutricionais e inflamação em pacientes renais crônicos tratados por hemodiálise [dissertação]. Botucatu: Universidade Estadual Paulista, Faculdade de Medicina de Botucatu; 2008.

12. Daugirdas JT. Second generation logarithmic estimates or single pool variable volume kt/V: an analysis of error. J Am Soc Nephrol. 1993;4(5):1205-13.

13. National Kidney Foundation. K/DOQI clinical practice guidelines for chronic kidney disease: evaluation, classification, and stratification. Am J Kidney Dis. 2002;39(2 Suppl 1):S1-266.

14. World Health Organization. Physical status: the use and interpretation of anthropometry. Geneva: WHO; 1995.

15. Lipschitz DA. Screening for nutritional status in the elderly. Prim Care. 1994;21(1):55-67.

16. Lohman TG, Roche AF, Martorell R, editors. Anthropometric standardization reference manual: abridged edition. Champaign: Human Kinetics Books; 1991.

17. Frisancho AR. Anthropmetric standards for the assessment of grwth and Nutritional status. Ann Arbor: The University of Michigan Press; 1990. https://doi.org/10.3998/mpub.12198
18. Frisancho AR. New norms of upper limb fat and muscle áreas for assessment of nutritional status. Am J Clin Nutr. 1981;34(11): 2540-5.

19. Roriz AK, de Oliveira CC, Moreira PA, Eickemberg M, Medeiros JM, Sampaio LR. Methods of predicting visceral fat in Brazilian adults and older adults: a comparison between anthropometry and computerized tomography. Arch Latinoam Nutr. 2011;61(1): 5-12.

20. Studenski SA, Peters KW, Alley DE, Cawthon PM, McLean RR, Harris TB, Ferrucci L, Guralnik JM, Fragala MS, Kenny AM, Kiel DP, Kritchevsky SB, Shardell MD, Dam TT, Vassileva MT. The FNIH sarcopenia project: rationale, study description, conference recommendations, and final estimates. J Gerontol A Biol Sci Med Sci. 2014;69(5):547-58. https://doi.org/10.1093/gerona/glu010

21. Biavo BMM, Martins CTB, Cunha LM, Araújo ML, Ribeiro MMC, Sachs A, Uezima CBB, Draibe SA, Rodrigues CIS, Barros EJG. Aspectos nutricionais e epidemiológicos de pacientes com doença renal crônica submetidos a tratamento hemodialítico no Brasil, 2010. J Bras Nefrol. 2012;34(3):206-15. https://doi.org/10.5935/01012800.20120001

22. Sesso RC, Lopes AA, Thomé FS, Lugon JR, Martins CT. Inquérito Brasileiro de Diálise Crônica 2014. J Bras Nefrol. 2016;38(1): 54-61.

23. Dumler F. A low phase angle is a marker of decreased nutritional status and increased mortality in chronic dialysis patients. Asaio. 2006;52(2):72A. https://doi.org/10.1097/00002480-20060300000299

24. Dantas MAS. Investigação de associações da força de preensão manual medida por dinamômetro com o estado nutricional e a sobrevida de pacientes iniciando hemodiálise de manutenção: estudo PROHEMO [tese]. Salvador (BA): Universidade Federal da Bahia; 2014.

25. Oliveira CMC. Marcadores nutricionais, inflamatórios e bioipedancia elétrica e sua associação com morbi-mortalidade em hemodiálise [tese]. Natal (RN): Universidade Federal do Rio Grande do Norte; 2010.

26. Inda Filho AJ, Melamed ML. Vitamina D e doença renal. O que nós sabemos e o que nós não sabemos. J Bras Nefrol. 2013;35(4): 323-31. https://doi.org/10.5935/0101-2800.20130051

27. Bucharles S, Barberato SH, Stinghen AE, Gruber B, Piekala L, Dambiski AC, Custodio MR, Pecoits-Filho R. Impact of cholecalciferol treatment on biomarkers of inflammation and myocardial structure in hemodialysis patients without hyperparathyroidism. J Ren Nutr. 2012;22(2):284-91. https://doi.org/10.1053/j.jrn.2011.07.001

28. Nishimura S, Manabe I, Nagai R. Adipose tissue inflammation in obesity and metabolic syndrome. Discov Med. 2009;8(41): $55-60$.

29. Chazot C, Laurent G, Charra B, Blanc C, VoVan C, Jean G, Vanel T, Terrat JC, Ruffet M. Malnutrition in long term hemodialysis survivor. Nephrol Dial Transplant. 2001;16(1):61-9. https://doi.org/10.1093/ ndt/16.1.61

30. Leal VO, Mafra D, Fouque D, Anjos LA. Uso da força de preensão manual na avaliação da função muscular de pacientes com doença renal crônica em diálise: uma revisão sistemática. Nephrol Dial Transplant. 2011;26:1354-60. https://doi.org/10.1093/ndt/ gfq487 